\title{
Robust control of the boost-pressure of a turbocharger subject to model uncertainty and disturbances
}

\author{
Tobias Posielek ${ }^{\dagger}$, Kai Wulff ${ }^{\ddagger * *}$ and Johann Reger ${ }^{\ddagger}$
}

\begin{abstract}
Controller design for the boost-pressure of an exhaust-gas turbo-charger is considered. To this end, we propose a sliding mode controller with integral action in the slidingvariable. The design is simple, requires a small number of sensors and is robust with respect to various uncertainties and disturbances. Compared to standard integral sliding-mode controllers our design approach yields a dynamics of lower order which simplifies the stability and invariance analysis.
\end{abstract}

\section{INTRODUCTION}

The use of turbochargers increases efficiency and reduces emissions of modern combustion engines. Turbocharged combustion engines are widely applied in a broad field of applications. This includes building and agricultural machinery, locomotive and ship engines and also modern power systems, like block thermal power plants or gas turbine systems and, of course, automotive systems. This may be the reason for the sustained interest in research on the control of turbochargers, see [1], [2], [3], [4], [5], [6], [7] to name but a few.

A schematic for the airpath of a typical combustion engine is shown in Fig. 1. The exhaust-gas of the combustion engine on the right drives a turbine which is connected with a compressor on the fresh air side of the airpath. The compressor increases the air pressure in the intake manifold and thus enhances its capability of carrying oxygen to the combustion. In order to manipulate the power of the compressor, the turbine is equipped with a so-called variable-turbine geometry (VTG) which allows to vary the effective area of the turbine. The control task considered in this contribution is to control the boost-pressure in the intake manifold using the VTG as actuator.

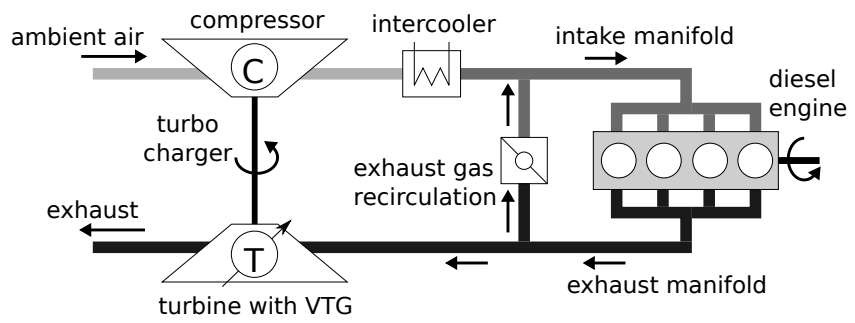

Fig. 1. Schematic of the airpath with turbocharger

Physical modeling and parameter-identification of a turbocharger system is a difficult and time-consuming task

\footnotetext{
†Author is with the Institute of Robotics and Mechatronics, German Aerospace Center (DLR), Muenchner Str. 20, D-82234 Wessling, Germany

$\ddagger$ Authors are with the Control Engineering Group, Technische Universität Ilmenau, P.O. Box 1005 65, D-98684, Ilmenau, Germany

${ }^{*}$ Corresponding author: kai.wulffetu-ilmenau. de
}

[8],[9],[10]. Due to its thermodynamics nature the process exhibits strong nonlinearities. Furthermore, the system parameters also depend on the construction of the airpath and the combustion engine. Therefore, the parameter identification of such systems typically requires large experimental effort and is prone to significant uncertainties. Furthermore, process information at runtime is often restricted to a few sensor signals.

In this contribution we consider the control of the boostpressure subject to model uncertainties and disturbances. In particular we shall consider constant parameter uncertainties and time-varying input disturbances. In our case-study, only measurements of the boost-pressure $p_{i}$ and the exhaust-gas pressure $p_{x}$ are available for the control.

Uncertainties and disturbances are called matched if they are within the input-span [11], otherwise they are called unmatched. Most parameter uncertainties in the airpath cause both types of uncertainties. It is well known that sliding-mode controllers may be successful in compensating (bounded) matched uncertainties. Unmatched uncertainties, in general, cannot be compensated by simple sliding-mode techniques [12]. However, with integral sliding-mode its influence can be minimised [11],[13],[14].

One of the first studies on the boost-pressure control using sliding-mode techniques is published in [15]. The authors devise a first-order sliding-mode controller combined with a state-observer. Attractivity of the sliding-manifold is guaranteed by the reaching law, however, there is no formal proof of stability of the dynamics on the sliding-manifold. In [16], [17] a MIMO approach is considered. Two control inputs, namely the exhaust-gas recirculation and VTG, are used to control the mass flow in the input-manifold as well as the exhaust-gas pressure as proposed in [18]. To the best knowledge of the authors, a study dedicated to the impact of various uncertainties for the boost-pressure control is not available to date.

Our approach is to use the VTG-actuator only and build our control design on the measurements of the boost-pressure and exhaust-gas pressure. We choose a sliding-manifold with integral action similar to the PID sliding-surface considered in [19] with some important modifications. The reduced dynamics of our approach are of third-order which allows to handle the stability analysis more easily. Classical integral sliding-mode techniques [13], [14] with an integrator in the nominal control yield a fourth-order dynamics on the sliding manifold, rendering the stability problem more complex.

The paper is organized as follows. In the subsequent section we recall the well-known third-order model of the 
airpath that shall be subject of our investigations. In Section III we propose our controller. We describe the system in regular form and choose a sliding-manifold with integral action on $p_{i}$. The reduced dynamics on the sliding-manifold is of third order. Stability of the closed-loop system is guaranteed by a quadratic Lyapunov function. In Section IV we highlight the relation to similar sliding-mode techniques like PID-sliding manifold design proposed in [19] or integral sliding-mode in [13], [14]. Section V presents simulation results of the proposed controller in comparison to two other control approaches. We investigate the control performance for matched and unmatched uncertainties. Conclusions are drawn in Section VI.

\section{MODEL OF THE AIRPATH}

Subject of our analysis is the well-established model for the airpath in [20], [18] with parameters given in [4]. The third-order model is derived by considering the mass-flow balances in the respective volumes, expressed by

$$
\begin{aligned}
\dot{p}_{i} & =\frac{R T_{i}}{V_{i}}\left(W_{c i}+W_{x i}-W_{i e}\right) \\
\dot{p}_{x} & =\frac{R T_{x}}{V_{x}}\left(W_{i e}+W_{f}-W_{x i}-W_{x t}\right) \\
\dot{P}_{c} & =\frac{1}{\tau}\left(-P_{c}+\eta_{m} P_{t}\right)
\end{aligned}
$$

where $p_{i}$ denotes the boost-pressure in the input manifold, $p_{x}$ the pressure in the output manifold and $P_{c}$ the power of the compressor. $W_{c i}$ is the flow rate from the compressor to the input manifold. $W_{x i}$ is the flow rate from the output manifold to the input manifold and describes the exhaust gas recirculation. $W_{i e}$ is the flow rate from the input manifold into the engine and $W_{x t}$ is the flow rate from the output manifold to the turbine.

The flow rates and the turbine power $P_{t}$ can be determined as in [21]. They are given by

$$
\begin{aligned}
W_{c i} & =\frac{\eta_{c}}{c_{p} T_{a}} \frac{P_{c}}{\left(\frac{p_{i}}{p_{x}}\right)^{\mu}-1}, \\
W_{x i} & =\frac{A_{e g r}\left(u_{e g r}\right) p_{x}}{\sqrt{R T_{x}}} \sqrt{\frac{2 p_{i}}{p_{x}}\left(1-\frac{p_{i}}{p_{x}}\right)} \\
W_{i e} & =\eta_{v} \frac{p_{i} n_{m} V_{d}}{2 R T_{i}}, \\
W_{x t} & =\left(c_{1} u_{v t g}+c_{2}\right)\left(c_{3}\left(\frac{p_{a}}{p_{x}}-1\right)+c_{4}\right) \\
P_{t} & =W_{x t} c_{p} T_{x} \eta_{t}\left(1-\left(\frac{p_{x}}{p_{r}} \sqrt{\frac{p_{r}}{p_{x}}}\right)^{\mu}\right) \cdot \sqrt{\frac{2 p_{i}}{p_{x}}\left(1-\frac{p_{a}}{p_{x}}\right)},
\end{aligned}
$$

The actuating variable is the VTG-position $u_{v t g}$ which influences the turbine flow rate $W_{x t}$. In this paper, we shall consider the nominal case where no exhaust gas recirculation is considered, i.e. $W_{x i}=0$. The systems states are required to remain in an admissible region given by:

$$
p_{i} \in\left[p_{a}, 3 \cdot 10^{5} \mathrm{~Pa}\right], p_{x} \in\left[p_{a}, 6 \cdot 10^{5} \mathrm{~Pa}\right], P_{c} \in[0,15 \mathrm{~kW}] .
$$

TABLE I

MODELPARAMETERS FROM [4]

\begin{tabular}{|c|l|c|c|}
\hline & Description & Value & $\mathrm{Unit}$ \\
\hline$n_{m}$ & Engine speed & 41.66 & {$\left[\mathrm{~s}^{-1}\right]$} \\
$W_{f}$ & Mass-flow fuel & $2.5 \mathrm{e}-3$ & {$\left[\mathrm{kgs}^{-1}\right]$} \\
$W_{c i}$ & Mass-flow compressor & - & {$\left[\mathrm{kgs}^{-1}\right]$} \\
$W_{x i}$ & Mass-flow EGR & - & {$\left[\mathrm{kgs}^{-1}\right]$} \\
$W_{i e}$ & Mass-flow intake manifold & - & {$\left[\mathrm{kgs}^{-1}\right]$} \\
$W_{x t}$ & Fuel rate exhaust manifold & - & {$\left[\mathrm{kgs}^{-1}\right]$} \\
$R$ & Specific gas constant & 287 & {$\left[\mathrm{Jkg}^{-1} \mathrm{~K}^{-1}\right]$} \\
$p_{i}$ & Intake manifold pressure & - & {$[\mathrm{Pa}]$} \\
$p_{x}$ & Exhaust manifold pressure & - & {$[\mathrm{Pa}]$} \\
$p_{r}$ & Reference pressure & $1.013 \mathrm{e} 5$ & {$[\mathrm{~Pa}]$} \\
$p_{a}$ & Ambient pressure & $1.013 \mathrm{e} 5$ & {$[\mathrm{~Pa}]$} \\
$P_{c}$ & Compressor power & - & {$[\mathrm{kW}]$} \\
$P_{t}$ & Turbine power & - & {$[\mathrm{kW}]$} \\
$T_{i}$ & Intake manifold temperature & 313 & {$[\mathrm{~K}]$} \\
$T_{x}$ & Exhaust manifold temperature & 509 & {$[\mathrm{~K}]$} \\
$T_{r}$ & Reference temperature & 298 & {$[\mathrm{~K}]$} \\
$T_{a}$ & Ambient temperature & 298 & {$[\mathrm{~K}]$} \\
$V_{i}$ & Intake manifold volume & 0.006 & {$\left[\mathrm{~m}{ }^{3}\right]$} \\
$V_{x}$ & Exhaust manifold volume & 0.001 & {$\left[\mathrm{~m}{ }^{3}\right]$} \\
$V_{d}$ & Displacement volume & 0.002 & {$\left[\mathrm{~m}{ }^{3}\right]$} \\
$\eta_{m}$ & Turbo mechanical efficiency & 98 & {$[\%]$} \\
$\eta_{c}$ & Compressor isentropic efficiency & 61 & {$[\%]$} \\
$\eta_{t}$ & Turbine isentropic efficiency & 76 & {$[\%]$} \\
$\eta_{v}$ & Volumetric efficiency & 87 & {$[\%]$} \\
$c_{p}$ & Specific heat at constant pressure & 1014.4 & {$\left[\mathrm{Jkg}^{-1} \mathrm{~K}-1\right]$} \\
$\mu$ & Constant & 0.286 & {$[-]$} \\
$\tau$ & Time-constant of turbocharger & 0.11 & {$[\mathrm{~s}]$} \\
$c_{1}$ & VTG Parameter & 0.136 & {$[-]$} \\
$c_{2}$ & VTG Parameter & 0.4 & {$[-]$} \\
$c_{3}$ & VTG Parameter & 0.6 & {$[-]$} \\
$c_{4}$ & VTG Parameter & & \\
& & & \\
\hline
\end{tabular}

The complete set of parameters is listed in Table I.

\section{CONTROL DESIGN}

For the control design we consider the system in regular form and choose a sliding-surface with integral action that will compensate stationary effects of unmatched disturbances. Suitable initialisation of the integrator state allows to eliminate the reaching phase such that the system is in the sliding phase at all times. The resulting reduced dynamics on the sliding-manifold is of third order. Stability of the reduced dynamics is guaranteed by a quadratic Lyapunov function.

\section{A. Regular form and disturbances}

Neglecting the actuator dynamics we shall consider the exhaust-gas mass flow $W_{x t}$ as input variable denoted by $u=$ $W_{x t}$ in the remainder of this contribution. For the design of a sliding-mode controller it is convenient to consider the system in the so-called regular form proposed in [22] and [23]. Following the assessment in [24] we introduce the new state

$$
z:=P_{c}+\frac{\delta}{\zeta} \int_{p_{a}}^{p_{x}} \Omega(p) d p
$$

where

$$
\delta:=\frac{\eta_{m} c_{p} T_{x} \eta_{t}}{\tau}, \quad \zeta:=\frac{R T_{x}}{V_{x}}, \quad \Omega\left(p_{x}\right):=1-\left(\frac{p_{a}}{p_{x}}\right)^{\mu} .
$$


With further abbreviations

$$
\begin{aligned}
\alpha & :=\frac{V_{d} \eta_{i}}{2 V_{i}}, \quad \beta:=\frac{R T_{i} \eta_{c}}{V_{i} c_{p} T_{a}}, \quad \gamma:=\frac{\eta_{V} V_{d} T_{x}}{2 V_{x} T_{i}} \\
\Phi\left(p_{i}\right) & :=\frac{\beta}{\left(\frac{p_{i}}{p_{a}}\right)^{\mu}-1}, \quad \Psi\left(p_{x}\right):=\frac{\delta}{\zeta} \int_{p_{a}}^{p_{x}} \Omega(p) d p,
\end{aligned}
$$

we obtain the system in regular form:

$$
\begin{aligned}
\dot{p}_{i} & =-\alpha n_{m} p_{i}+\Phi\left(p_{i}\right)\left(z-\Psi\left(p_{x}\right)\right) \\
\dot{z} & =\frac{\delta}{\zeta} \Omega\left(p_{x}\right)\left(\gamma n_{m} p_{i}+\zeta W_{f}\right)-\frac{1}{\tau}\left(z-\Psi\left(p_{x}\right)\right) \\
\dot{p}_{x} & =\gamma n_{m} p_{i}+\zeta W_{f}-\zeta u .
\end{aligned}
$$

Note that $\Omega\left(p_{x}\right) \in[0,1]$ and $\Omega$ and $\Psi$ are monotonically increasing functions, while $\Phi$ is monotonically decreasing and unbounded for $p_{i} \rightarrow p_{a}$. For each setpoint $p_{i}^{*}$ of the system (11)-(13) there exists a unique equilibrium $\left(p_{i}^{*}, z^{*}, p_{x}^{*}\right)$ and corresponding stationary control $u^{*}$. The admissible region (9) transformed into the new coordinates $\left(p_{i}, z, p_{x}\right)$ shall be denoted by $\mathbb{X}$.

For the turbo-charger system in regular form (11)-(13), unmatched uncertainties enter the system in equation (11) or (12), whereas the matched disturbance adds to the right-hand side of (13), that is

$$
\begin{aligned}
\dot{p}_{i} & =-\alpha n_{m} p_{i}+\Phi\left(p_{i}\right)\left(z-\Psi\left(p_{x}\right)\right)+\phi_{u 1} \\
\dot{z} & =\frac{\delta}{\zeta} \Omega\left(p_{x}\right)\left(\gamma n_{m} p_{i}+\zeta W_{f}\right)-\frac{1}{\tau}\left(z-\Psi\left(p_{x}\right)\right)+\phi_{u 2} \\
\dot{p}_{x} & =\gamma n_{m} p_{i}+\zeta W_{f}-\zeta u+\phi_{m}
\end{aligned}
$$

where $\phi_{m}$ denotes the matched and $\phi_{u 1}, \phi_{u 2}$ the unmatched disturbance. The unmatched disturbance is state-dependent, the matched disturbance may be state and/or time-dependent.

In a practical application, we expect to face both types of disturbances. For instance, a parameter uncertainty of $c_{2}$ in (7) will result in some matched disturbance $\phi_{m}$, whereas inspection of the system in regular form (11)-(13) reveals that most other parameter uncertainties lead to an unmatched uncertainty $\phi_{u 1}$ or $\phi_{u 2}$ or a combination of both. One of our major concerns are uncertainties that do not vanish at the nominal equilibrium point. Thus, the equilibrium may change due to the uncertainty. These kind of uncertainties may prohibit that the boost-pressure of the closed loop system converges to the set-point. We propose a control law that drives the system to its setpoint despite the presence of such uncertainties and also compensates the matched (possibly time-varying) disturbance.

\section{B. Proposed sliding-mode controller}

Sliding mode control ensures robustness to all bounded matched uncertainties. In order to address unmatched uncertainties which affect the stationary value of the boostpressure, an additional integrator state can be introduced and used in the control law.

The idea of choosing a PID-type sliding-surface goes back to [25] and [19] and has been extended and applied in various applications, see e.g. [26], [27] and [28]. Adopted for our system such sliding-surface takes the form:

$$
\begin{aligned}
s & =k_{p}\left(p_{i}-p_{i}^{*}\right)+k_{i} v+k_{d} \frac{d}{d t}\left(p_{i}-p_{i}^{*}\right) \\
& =k_{p}\left(p_{i}-p_{i}^{*}\right)+k_{i} v+k_{d}\left(-\alpha n_{m} p_{i}+\Phi\left(p_{i}\right)\left(z-\Psi\left(p_{x}\right)\right)\right)
\end{aligned}
$$

with $\dot{v}=p_{i}-p_{i}^{*}$. The control law is typically obtained by requiring $\dot{s}=0$. Note, that the derivative-part $k_{d}$ in $s$ is essential in order to obtain the control input $u$ in $\dot{s}$.

Inspection of the equations above reveals two drawbacks of this approach for our application scenario: Firstly, the control law derived from the second equation will contain the state $z$ and thus the measurement $P_{c}$, c.f. (10), which is not available; secondly, we need to take the derivative of the signal $p_{i}$. This can cause additional problems in the presence of measurement noise.

Therefore we choose a much simpler sliding-surface and incorporate a second state $p_{x}$ :

$$
\begin{aligned}
& s=p_{x}-p_{x}^{*}-v \\
& \dot{v}=-k\left(p_{i}-p_{i}^{*}\right), \quad v(0)=v_{0},
\end{aligned}
$$

with $k>0$. Note that additional design parameters in $s$ do not increase the freedom in shaping the reduced dynamics. Consequently, we choose the control law as the superposition of the equivalent control and a discontinuous control [12]:

$$
u=\frac{1}{\zeta}\left(\gamma n_{m} p_{i}+\zeta W_{f}+k\left(p_{i}-p_{i}^{*}\right)+\rho \operatorname{sgn}(s)\right) .
$$

The choice of $\rho>\left|\phi_{m}\right|$ for all times guarantees the attractivity of the sliding manifold $s=0$.

On the sliding-manifold $s=0$ holds $v=p_{x}-p_{x}^{*}$ and thus $\dot{p}_{x}=-k\left(p_{x}-p_{x}^{*}\right)$. From (11)-(13) we obtain the reduced dynamics

$$
\begin{aligned}
\dot{p}_{i} & =-\alpha n_{m} p_{i}+\Phi\left(p_{i}\right)\left(z-\Psi\left(p_{x}\right)\right) \\
\dot{z} & =\frac{\delta}{\zeta} \Omega\left(p_{x}\right)\left(\gamma n_{m} p_{i}+\zeta W_{f}\right)-\frac{1}{\tau}\left(z-\Psi\left(p_{x}\right)\right) \\
\dot{p}_{x} & =-k\left(p_{i}-p_{i}^{*}\right) .
\end{aligned}
$$

If the sliding-manifold is attractive and the reduced dynamics is asymptotically stable, at the equilibrium we have

$$
\dot{p}_{x}=0 \Rightarrow p_{i}=p_{i}^{*}
$$

Note that this last result is robust against any unmatched disturbance $\phi_{u 1}, \phi_{u 2}$. Matched disturbances $\phi_{m}$ are fully compensated if $\rho>\left|\phi_{m}\right|$.

\section{Stability analysis}

By choosing $v_{0}=p_{x}(0)-p_{x}^{*}(0)$ we can ensure that the trajectory starts on the sliding manifold. Thus, asymptotic stability of the reduced dynamics (20)-(22) guarantees stability of the closed-loop system (11)-(13) and (19).

Consider the nominal case, i.e. $\phi_{u 1}=\phi_{u 2}=\phi_{m}=0$. We show stability and determine a part of the region of attraction using a quadratic Lyapunov function that we draw 
from the linearised system. Linearisation at the equilibrium $\left(p_{i}^{*}, z^{*}, p_{x}^{*}\right)$ yields the dynamics matrix

$$
A=\left[\begin{array}{cc}
-\alpha n_{m}+\frac{d}{d p_{i}} \Phi\left(p_{i}^{*}\right)\left(z^{*}-\Psi\left(p_{x}^{*}\right)\right) & \Phi\left(p_{i}^{*}\right) \\
\frac{\delta}{\zeta} \Omega\left(p_{x}^{*}\right) \gamma n_{m} & -\frac{1}{\tau} \\
-k & 0 \\
-\Phi\left(p_{i}^{*}\right) \frac{\delta}{\zeta} \Omega\left(p_{x}^{*}\right) \\
0
\end{array}\right] .
$$

An estimate of the region of attraction for the nonlinear system is obtained by the largest admissible level set for which $x^{\mathrm{T}} \operatorname{Pf}(x)<0$, where $x=\left(p_{i}, z, p_{x}\right)^{\mathrm{T}} . f$ denotes the right-hand side of (20)-(22), and $P$ is symmetric and positive definite solution of the corresponding Lyapunov equation.

It turns out that the level-sets tend to span only a small interval in the $p_{x}$-direction. Therefore we formulate the following optimisation problem:

$$
\min _{q_{2}, q_{3}>0}-p_{x_{\mathrm{opt}}}\left(q_{2}, q_{3}\right)
$$

subject to

$$
\begin{gathered}
A^{\mathrm{T}} P+P A=-\left(\begin{array}{ccc}
1 & 0 & 0 \\
0 & q_{2} & 0 \\
0 & 0 & q_{3}
\end{array}\right), \\
\Omega_{c}=\left\{x \in \mathbb{R}^{3} \mid x^{\mathrm{T}} P x=c\right\}, \\
c_{\max }=\max \left\{c \in \mathbb{R}^{+} \mid \forall x \in \Omega_{c} \cap \mathbb{X}: x^{\mathrm{T}} \operatorname{Pf}(x)<0\right\}, \\
p_{x_{\mathrm{opt}}}\left(q_{2}, q_{3}\right)=\max \left\{p_{x} \in \mathbb{R}^{+} \mid p_{x} \in \Omega_{c_{\max }}\right\} .
\end{gathered}
$$

For the setpoint $p_{i}^{*}=2000$ the determined region of attraction is shown in Fig. 2. The outlined shape depicts the admissible region of safe operation of the turbo-charger. While the determined level-set is not a strict subset of the admissible region $\mathbb{X}$, we can verify numerically via a simple invariance condition that the intersection of the admissible region and this level set is positively invariant. This guarantees that the intersection is part of the region of attraction of the equilibrium point.



Fig. 2. Estimated region of attraction and invariant admissible region $\mathbb{X}$

\section{RELATION TO INTEGRAL SLIDING-MODE}

The proposed control design belongs to the class of integral sliding-mode (ISM) controllers defined in [11]. In recent years a design procedure has been proposed that extends (any) nominal control law $u_{0}$ by a discontinuous control $u_{1}$, minimising the impact of the unmatched uncertainty, [13], [29], [14]. In regard to our problem (11)-(13), the nominal control is to address the unmatched uncertainty whereas the integral sliding-mode controller eliminates the matched uncertainty. In the following we shall design such a ISM control and cast our proposed controller into this framework.

\section{A. Classical design}

In order to compensate time-invariant unmatched uncertainties, we may choose a PI-state-feedback controller for the nominal control $u_{0}$, that is

$$
\begin{aligned}
\dot{v} & =p_{i}-p_{i}^{*} \\
u_{0} & =k_{s f}^{\mathrm{T}}\left(\begin{array}{lll}
p_{i} & z & p_{x}
\end{array}\right)^{\mathrm{T}}+k_{i} v,
\end{aligned}
$$

where $k_{s f} \in \mathbb{R}^{3}$. Following [13], [14], optimal disturbance rejection is achieved choosing the sliding manifold:

$$
s=p_{x}-p_{x}^{*}+v_{2} .
$$

This leads to integrator state and discontinuous dynamics

$$
\begin{aligned}
\dot{v}_{2} & =-\left(\gamma n_{m} p_{i}+\zeta W_{f}-\zeta u_{0}\right), \\
u_{1} & =\frac{\rho}{\zeta} \operatorname{sgn}(s) .
\end{aligned}
$$

On the sliding surface we obtain the reduced dynamics

$$
\begin{aligned}
\dot{p}_{i} & =-\alpha n_{m} p_{i}+\Phi\left(p_{i}\right)\left(z-\Psi\left(p_{x}\right)\right) \\
\dot{z} & =\frac{\delta}{\zeta} \Omega\left(p_{x}\right)\left(\gamma n_{m} p_{i}+\zeta W_{f}\right)-\frac{1}{\tau}\left(z-\Psi\left(p_{x}\right)\right) \\
\dot{p}_{x} & =\gamma n_{m} p_{i}+\zeta W_{f}+k_{s f}^{\mathrm{T}}\left(p_{i} \quad z \quad p_{x}\right)^{\mathrm{T}}+k_{i} v \\
\dot{v} & =p_{i}-p_{i}^{*} .
\end{aligned}
$$

When constraint to the sliding-surface, the system behaves just like the nominal closed-loop system with PI-statefeedback control. Assuming a suitable design, the matched uncertainty has no impact and the unmatched time-invariant uncertainty does not influence the stationary behaviour of the input pressure. However, the reduced dynamics is of fourthorder as compared to the third-order dynamics (20)-(22) of our proposed approach. This renders the stability analysis much more complex.

\section{B. Proposed design in form of classical ISM}

The resulting dynamics of the classical ISM design is completely defined by the ideal control $u_{0}$ and the choice of the switching function in the presence of unmatched uncertainties. Thus, ISM can be seen as an addition to an existing control which ensures robustness to matched uncertainties. With regard to unmatched uncertainties, this means that the ISM control does not excess the performance of the control $u_{0}$ alone.

We have chosen a different approach to design the ISM controller. Instead of minimising the effect of the unmatched 
uncertainty in comparison to the ideal control, we use the switching function to shape the reduced dynamics. The integrator state $v$ is used to ensure the stationary accuracy of the boost-pressure which, however, reduces the design freedom for the ideal control $u_{0}$.

If we cast our approach into the classical design we obtain:

$$
\begin{aligned}
u_{0} & =\frac{1}{\zeta}\left(\gamma n_{m} p_{i}+\zeta W_{f}+k\left(p_{i}-p_{i}^{*}\right)\right), \\
s & =p_{x}-p_{x}^{*}+v, \\
u_{1} & =\frac{\rho}{\zeta} \operatorname{sgn}(s), \\
\dot{v} & =k\left(p_{i}-p_{i}^{*}\right) .
\end{aligned}
$$

In light of this, our proposed control can be written in the sense of [14]. Our choice of the sliding manifold also minimises the effect of unmatched disturbances as proven in [13]. At a first glance, it might seem remarkable that $u_{0}$ does not contain integral action. Substituting $u_{0}$ into (13) reveals that $p_{x}$ essentially serves this duty. A different interpretation may be that the integrator $v$ of the sliding surface doubles up as integrator for the nominal control.

\section{Simulation RESUlts}

Simulation results shall illustrate the performance of our controller. We consider a time-invariant unmatched uncertainty and also a time-varying matched uncertainty. In order to highlight the robustness properties, we also design a simple PI state-feedback controller (capable of compensating constant model uncertainties) and a first-order sliding-mode controller (capable of compensating matched uncertainties). The PI state-feedback of the form (24) is devised such that the eigenvalues of linearised closed-loop dynamics are $\{-21,-21,-2,-2\}$. The sliding-surface and control law of the first-order SMC is obtained from (17), (18) and (19) with $k=0, \rho=2 \times 10^{6}$. In order to reduce chattering, we approximate the discontinuity $\operatorname{sgn}(s) \approx \frac{s}{|s|+1000}$. For our proposed controller we choose $k=7, \rho=2 \times 10^{6}$.

Note that the three controllers are not tuned for best performance, but to give similar rise and settling times to allow for a better comparison.

If proved to be stable, using the classical ISM discussed in Section IV-A we would expect a similar performance compared to our proposed approach.

We simulate the system with the proposed controllers under the influence of different parameter uncertainties and evaluate the results. In the first scenario, we consider the parameter $c_{2}$ to be uncertain by $10 \%$ and time-varying so that a matched disturbance affects the system (14)-(16) with:

$$
\phi_{m}=-\zeta \tilde{c}_{2} \sin (4 \pi t)\left(c_{3}\left(\frac{p_{a}}{p_{x}}-1\right)+c_{4}\right),
$$

with $\tilde{c}_{2}=0.1 c_{2}$.

For the second scenario, we admit a parameter uncertainty of $10 \%$ in $\gamma$. With $\tilde{\gamma}=0.1 \gamma$ this causes unmatched and matched uncertainties given by:

$$
\phi_{u 1}=0, \quad \phi_{u 2}=\frac{\delta}{\zeta} \Omega\left(p_{x}\right) \tilde{\gamma} n_{m} p_{i}, \quad \phi_{m}=\tilde{\gamma} n_{m} p_{i} .
$$
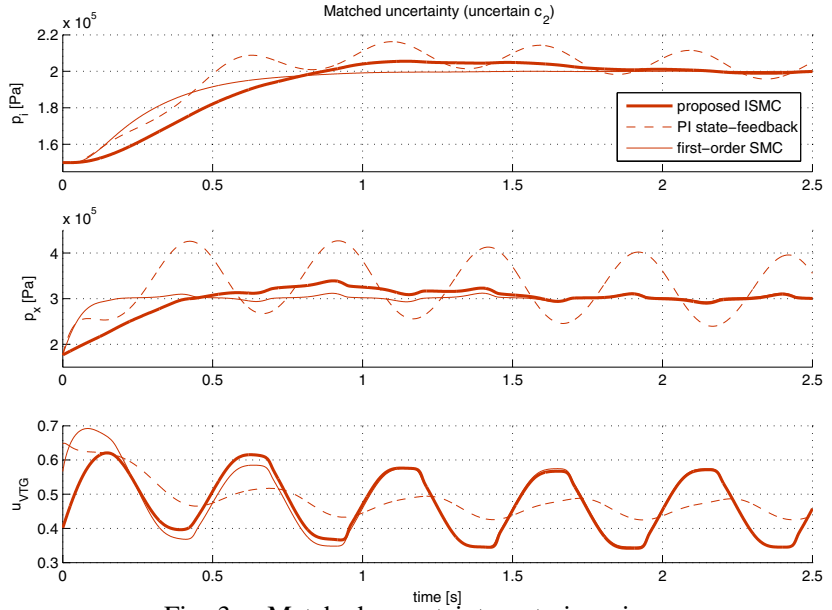

Fig. 3. Matched uncertainty entering via $c_{2}$
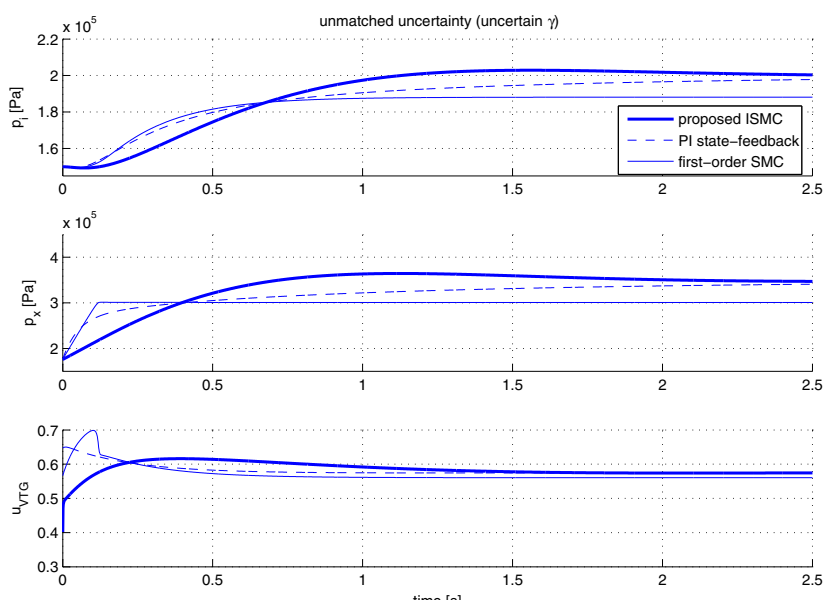

Fig. 4. Unmatched and matched uncertainty entering via $\gamma$

Fig. 3 shows the simulation results of the three controllers for the time-varying matched uncertainty $\phi_{m}$ in (29). The first two graphs show the states $p_{i}$ and $p_{x}$, respectively. The third plot shows the normalised control signal. The system with PI state-feedback does not reach steady state due to persistent disturbance. Both sliding-mode controllers can compensate the matched uncertainty.

In Fig. 4 we see the results for the second scenario (30) with the uncertainty in $\gamma$. The PI state-feedback can compensate the stationary influence of both uncertainties on the input pressure. The first-order SMC, however, shows an offset in the boost-pressure $p_{i}$ and the exhaust-gas pressure $p_{x}$. This drawback is not present for the proposed ISM controller.

Fig. 5 shows a comparison for each controller of the nominal undisturbed response and each of the two scenarios considered above. As expected, the two sliding-mode controllers show (almost) no differences to the nominal performance when a matched disturbance is added. However, our proposed ISM controller (2nd plot) shows the most consistent performance under uncertainties.

\section{CONCLUSION}

We have devised a controller for the boost-pressure control in an exhaust-gas turbocharger system under uncertainties. 

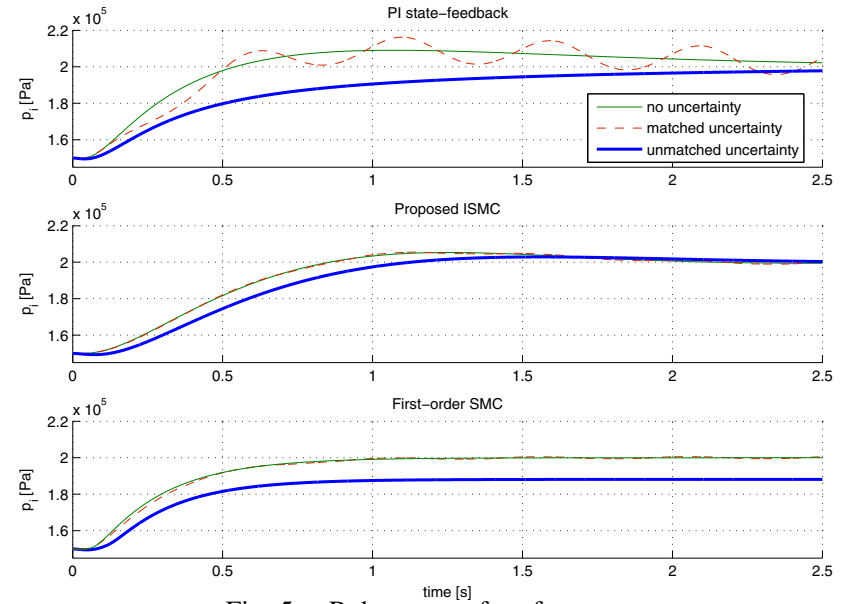

Fig. 5. Robustness of performance

Using the variable turbine geometry as the only control input, first, the system was transformed into regular form. Afterwards, the typical sliding mode design was augmented by an integrator to deal with the stationary effect of the unmatched uncertainty. We show stability of the controlled system using Lyapunov stability theory. In a comparison with classical ISM we clarified the different purposes of the integral state. Whereas in classical ISM the integrator state mainly allows an immediate start in sliding mode while retaining the performance of the nominal control, our design uses the integrator state to reduce the influence of the unmatched uncertainty. Still both design concepts can be reformulated into each other. Simulation results show that the proposed controller is robust with respect to matched and unmatched uncertainties. Further work may concentrate on the design of the sliding manifold, incorporating all states to improve the performance while retaining the robustness properties.

\section{ACKNOWLEDGEMENT}

Johann Reger kindly acknowledges support by the European Union Horizon 2020 research and innovation program under Marie Skłodowska-Curie grant agreement No. 734832.

\section{REFERENCES}

[1] O. Flärdh, G. Ericsson, E. Klingborg, and J. Mårtensson, "Optimal air path control during load transients on a spark ignited engine with variable geometry turbine and variable valve timing," IEEE Transactions on Control Systems Technology, vol. 22, no. 1, pp. 83-93, 2014.

[2] N. E. Kahveci, S. T. Impram, and A. U. Genc, "Boost pressure control for a large diesel engine with turbocharger," in American Control Conference, 2014, pp. 2108-2113.

[3] H. Borhan, G. Kothandaraman, and B. Pattel, "Air handling control of a diesel engine with a complex dual-loop EGR and VGT air system using MPC," in American Control Conference, 2015, pp. 4509-4516.

[4] M. Tejada Zuñiga, M. Noack, and J. Reger, "Observer designs for a turbocharger system of a diesel engine," in Congreso Latinoamericano de Control Automático, 2016, pp. 354-361.

[5] M. Noack, K. Wulff, J. Reger, and M. H. Höper, "Observability analysis and nonlinear observer design for a turbocharger in a diesel engine," in Conference on Control Applications, 2016, pp. 323-328.

[6] K. Song, D. Upadhyay, H. Sun, H. Xie, and G. Zhu, "A physics-based control-oriented model for compressor mass flow rate," in Conference on Decision and Control, 2016, pp. 6733-6738.
[7] Y. Liu, J. Zhou, L. Fiorentini, M. Canova, and Y. Y. Wang, "Control of a two-stage turbocharged diesel engine air path system for mode transition via feedback linearization," in American Control Conference, 2016, pp. 5105-5111.

[8] P. Moraal and I. Kolmanovsky, "Turbocharger modeling for automotive control applications," in SAE Technical Paper, 03 1999, pp. 309-322.

[9] L. Guzzella and C. H. Onder, Introduction to Modeling and Control of Internal Combustion Engine Systems, 2nd ed. Springer, 2010.

[10] J. Wahlström and L. Eriksson, "Modelling diesel engines with a variable-geometry turbocharger and exhaust gas recirculation by optimization of model parameters for capturing non-linear system dynamics," Journal of Automobile Engineering, vol. 225, no. D, pp. 960-986, 2011.

[11] V. Utkin and J. Shi, "Integral sliding mode in systems operating under uncertainty conditions," in Conference on Decision and Control, 1996, pp. 4591-4596.

[12] Y. Shtessel, C. Edwards, L. Fridman, and A. Levant, Sliding Mode Control and Observation. Springer, 2014.

[13] F. Castaños and L. Fridman, "Analysis and design of integral sliding manifolds for systems with unmatched perturbations," IEEE Transactions on Automatic Control, vol. 51, no. 5, pp. 853-858, 2006.

[14] M. Rubagotti, A. Estrada, F. Castanos, A. Ferrara, and L. Fridman, "Integral sliding mode control for nonlinear systems with matched and unmatched perturbations," IEEE Transactions on Automatic Control, vol. 56, no. 11, pp. 2699-2704, 2011.

[15] V. I. Utkin, H.-C. Chang, I. Kolmanovsky, and J. A. Cook, "Sliding mode control for variable geometry turbocharged diesel engines," in American Control Conference, 2000, pp. 584-588.

[16] S. A. Ali, B. N'doye, and L. Nicolas, "Sliding mode control for turbocharged diesel engine," in Mediterranean Conference on Control Automation, 2012, pp. 996-1001.

[17] S. A. Ali and N. Langlois, "Sliding mode control for diesel engine air path subject to matched and unmatched disturbances using extended state observer," Mathematical Problems in Engineering, 2013.

[18] M. Jankovic, M. Jankovic, and I. Kolmanovsky, "Constructive Lyapunov control design for turbocharged diesel engines," IEEE Transactions on Control Systems Technology, vol. 8, no. 2, pp. 288-299, 2000.

[19] S. Seshagiri and H. K. Khalil, "On introducing integral action in sliding mode control," in Conference on Decision and Control, 2002, pp. 1473-1478.

[20] M. Jankovic, M. Jankovic, and I. Kolmanovsky, "Robust nonlinear control for turbocharged diesel engines," in American Control Conference, 1998, p. 13891394

[21] M. Schollmeyer, "Beitrag zur modellbasierten Ladedruckregelung für PKW-Dieselmotoren,” Ph.D. dissertation, Gottfried Wilhelm Leibniz Universität Hannover, 2010.

[22] A. G. Luk'yanov and V. I. Utkin, "Methods for reduction of equations of dynamic systems to a regular form," Automation and Remote Control, vol. 4, no. 1, pp. 5-13, 1981.

[23] W. Perruquetti, J. P. Richard, and P. Borne, "A generalized regular form for multivariable sliding mode control," Mathematical Problems in Engineering, vol. 7, no. 1, pp. 15-27, 2001.

[24] J. Schreibeis, K. Wulff, J. Reger, and J. A. Moreno, "Lyapunovstability for the sliding-mode control of a turbocharger subject to state constraints," in 43rd Annual Conference of the IEEE Industrial Electronics Society, 2017, [submitted].

[25] L.-W. Chang, "Dynamics of a sliding control with a first-order plus integral sliding condition," Dynamics and Control, vol. 2, pp. 201-219, 1992.

[26] I. Eker, "Sliding mode control with pid sliding surface and experimental application to an electromechanical plant," ISA transactions, vol. 45, no. 1, pp. 109-118, 2006.

[27] İ. Eker and Ş. A. Akınal, "Sliding mode control with integral augmented sliding surface: design and experimental application to an electromechanical system," Electrical Engineering, vol. 90, no. 3, pp. 189-197, 2008.

[28] M. Furat and İ. Eker, "Second-order integral sliding-mode control with experimental application," ISA transactions, vol. 53, no. 5, pp. 1661$1669,2014$.

[29] M. Rubagotti, A. Estrada, F. Castanos, A. Ferrara, and L. Fridman, "Optimal disturbance rejection via integral sliding mode control for uncertain systems in regular form," in Workshop on Variable Structure Systems, 2010, pp. 78-82. 